\title{
Sharp Bounds for Toader Mean in terms of Arithmetic and Second Contraharmonic Means
}

\author{
Wei-Mao Qian, ${ }^{1}$ Ying-Qing Song, ${ }^{2}$ Xiao-Hui Zhang, ${ }^{2}$ and Yu-Ming Chu ${ }^{2}$ \\ ${ }^{1}$ School of Distance Education, Huzhou Broadcast and TV University, Huzhou 313000, China \\ ${ }^{2}$ School of Mathematics and Computation Science, Hunan City University, Yiyang 413000, China \\ Correspondence should be addressed to Yu-Ming Chu; chuyuming2005@126.com
}

Received 25 April 2015; Accepted 10 September 2015

Academic Editor: Lars E. Persson

Copyright (C) 2015 Wei-Mao Qian et al. This is an open access article distributed under the Creative Commons Attribution License, which permits unrestricted use, distribution, and reproduction in any medium, provided the original work is properly cited.

We present the best possible parameters $\lambda_{1}, \mu_{1} \in \mathbb{R}$ and $\lambda_{2}, \mu_{2} \in(1 / 2,1)$ such that double inequalities $\lambda_{1} C(a, b)+\left(1-\lambda_{1}\right) A(a, b)<$ $T(a, b)<\mu_{1} C(a, b)+\left(1-\mu_{1}\right) A(a, b), C\left[\lambda_{2} a+\left(1-\lambda_{2}\right) b, \lambda_{2} b+\left(1-\lambda_{2}\right) a\right]<T(a, b)<C\left[\mu_{2} a+\left(1-\mu_{2}\right) b, \mu_{2} b+\left(1-\mu_{2}\right) a\right]$ hold for all $a, b>0$ with $a \neq b$, where $A(a, b)=(a+b) / 2, C(a, b)=\left(a^{3}+b^{3}\right) /\left(a^{2}+b^{2}\right)$ and $T(a, b)=2 \int_{0}^{\pi / 2} \sqrt{a^{2} \cos ^{2} \theta+b^{2} \sin ^{2} \theta} d \theta / \pi$ are the arithmetic, second contraharmonic, and Toader means of $a$ and $b$, respectively.

\section{Introduction}

For $a, b>0$ the Toader mean $T(a, b)$ [1], second contraharmonic mean $C(a, b)$, and arithmetic mean $A(a, b)$ of $a$ and $b$ are given by

$$
\begin{aligned}
T(a, b) & =\frac{2}{\pi} \int_{0}^{\pi / 2} \sqrt{a^{2} \cos ^{2} \theta+b^{2} \sin ^{2} \theta} d \theta \\
& = \begin{cases}\frac{2 a \mathscr{E}\left(\sqrt{1-(b / a)^{2}}\right)}{\pi}, & a>b, \\
\frac{2 b \mathscr{E}\left(\sqrt{1-(a / b)^{2}}\right)}{\pi}, & a<b, \\
a, & a=b,\end{cases} \\
C(a, b) & =\frac{a^{3}+b^{3}}{a^{2}+b^{2}},
\end{aligned}
$$

respectively, where $\mathscr{E}(r)=\int_{0}^{\pi / 2}\left(1-r^{2} \sin ^{2} t\right)^{1 / 2} d t(r \in(0,1))$ is the complete elliptic integral of the second kind. The Toader mean $T(a, b)$ is well known in mathematical literature for many years; it satisfies

$$
\begin{aligned}
& T(a, b)=R_{E}\left(a^{2}, b^{2}\right), \\
& T(1, r)=\frac{2}{\pi} \mathscr{E}\left(\sqrt{1-r^{2}}\right)
\end{aligned}
$$

for all $a, b>0$ and $0<r<1$, where

$$
R_{E}(a, b)=\frac{1}{\pi} \int_{0}^{\infty} \frac{[a(t+b)+b(t+a)] t}{(t+a)^{3 / 2}(t+b)^{3 / 2}} d t
$$

stands for the symmetric complete elliptic integral of the second kind (see [2-4]); therefore it cannot be expressed in terms of the elementary transcendental functions.

Recently, the Toader mean $T(a, b)$ has been the subject of intensive research. In particular, many remarkable inequalities for the Toader mean can be found in the literature [5-9].

Let $p \in \mathbb{R}, q \in[0,1]$, and $a, b>0$. Then the $p$ th power mean $M_{p}(a, b)$, pth Gini mean $G_{p}(a, b)$, pth Lehmer 
mean $L_{p}(a, b)$, and $q$ th generalized Seiffert mean $S_{q}(a, b)$ are defined by

$$
\begin{aligned}
& M_{p}(a, b)= \begin{cases}\left(\frac{a^{p}+b^{p}}{2}\right)^{1 / p}, & p \neq 0, \\
\sqrt{a b}, & p=0,\end{cases} \\
& G_{p}(a, b)= \begin{cases}\left(\frac{a^{p-1}+b^{p-1}}{a+b}\right)^{1 /(p-2)}, & p \neq 2, \\
\left(a^{a} b^{b}\right)^{1 /(a+b)}, & p=2,\end{cases} \\
& L_{p}(a, b)=\frac{a^{p+1}+b^{p+1}}{a^{p}+b^{p},} \\
& S_{q}(a, b) \quad 0<p \leq 1, a \neq b, \\
& = \begin{cases}\frac{p(a-b)}{\arctan [2 p(a-b) /(a+b)]}, & a=b, \\
\frac{a+b}{2}, & 0=0, a \neq b, \\
a, & \end{cases}
\end{aligned}
$$

respectively. It is well known that $M_{p}(a, b), G_{p}(a, b), L_{p}(a, b)$, and $S_{q}(a, b)$ are continuous and strictly increasing with respect to $p \in \mathbb{R}$ and $q \in[0,1]$ for fixed $a, b>0$ with $a \neq b$, respectively.

Vuorinen [10] conjectured that inequality

$$
M_{3 / 2}(a, b)<T(a, b)
$$

holds for all $a, b>0$ with $a \neq b$. This conjecture was proved by Qiu and Shen [11] and Barnard et al. [12], respectively.

Alzer and Qiu [13] presented a best possible upper power mean bound for the Toader mean as follows:

$$
T(a, b)<M_{\log 2 /(\log \pi-\log 2)}(a, b)
$$

for all $a, b>0$ with $a \neq b$.

In $[14,15]$, the authors found the best possible parameters $\alpha, \beta \in[0,1]$ and $\lambda, \mu \in \mathbb{R}$ such that double inequalities $S_{\alpha}(a, b)<T(a, b)<S_{\beta}(a, b)$ and $G_{\lambda}(a, b)<T(a, b)<$ $G_{\mu}(a, b)$ hold for all $a, b>0$ with $a \neq b$.

Chu and Wang [16] proved that double inequality

$$
L_{p}(a, b)<T(a, b)<L_{q}(a, b)
$$

holds for all $a, b>0$ with $a \neq b$ if and only if $p \leq 0$ and $q \geq 1 / 4$.

Inequality (8) leads to

$$
\begin{aligned}
A(a, b) & =L_{0}(a, b)<T(a, b)<L_{1 / 4}(a, b)<L_{2}(a, b) \\
& =C(a, b)
\end{aligned}
$$

for all $a, b>0$ with $a \neq b$.

Let $a, b>0$ with $a \neq b$ be fixed and $f(x)=C[x a+(1-$ $x) b, x b+(1-x) a]$. Then it is not difficult to verify that $f(x)$ is continuous and strictly increasing on $[1 / 2,1]$. Note that

$$
f\left(\frac{1}{2}\right)=A(a, b)<T(a, b)<C(a, b)=f(1) .
$$

Motivated by inequalities (9) and (10), it is natural to ask what are the best possible parameters, $\lambda_{1}, \mu_{1} \in \mathbb{R}$ and $\lambda_{2}, \mu_{2} \in$ $(1 / 2,1)$, such that double inequalities

$$
\begin{aligned}
& \lambda_{1} C(a, b)+\left(1-\lambda_{1}\right) A(a, b)<T(a, b) \\
& \quad<\mu_{1} C(a, b)+\left(1-\mu_{1}\right) A(a, b) \\
& C\left[\lambda_{2} a+\left(1-\lambda_{2}\right) b, \lambda_{2} b+\left(1-\lambda_{2}\right) a\right]<T(a, b) \\
& \quad<C\left[\mu_{2} a+\left(1-\mu_{2}\right) b, \mu_{2} b+\left(1-\mu_{2}\right) a\right]
\end{aligned}
$$

hold for all $a, b>0$ with $a \neq b$ ? The main purpose of this paper is to answer this question.

\section{Main Results}

In order to prove our main results we need some basic knowledge and two lemmas, which we present in this section.

For $r \in(0,1)$ the complete elliptic integral $\mathscr{K}(r)$ of the first kind is defined by

$$
\mathscr{K}(r)=\int_{0}^{\pi / 2}\left(1-r^{2} \sin ^{2} t\right)^{-1 / 2} d t .
$$

We clearly see that

$$
\begin{aligned}
& \mathscr{K}\left(0^{+}\right)=\mathscr{E}\left(0^{+}\right)=\frac{\pi}{2}, \\
& \mathscr{E}\left(1^{-}\right)=1,
\end{aligned}
$$

and $\mathscr{K}(r)$ and $\mathscr{E}(r)$ satisfy formulas (see [17, Appendix E, p. 474-475])

$$
\begin{aligned}
\frac{d \mathscr{K}(r)}{d r} & =\frac{\mathscr{E}(r)-\left(1-r^{2}\right) \mathscr{K}(r)}{r\left(1-r^{2}\right)}, \\
\frac{d \mathscr{E}(r)}{d r} & =\frac{\mathscr{E}(r)-\mathscr{K}(r)}{r}, \\
\mathscr{E}\left(\frac{2 \sqrt{r}}{1+r}\right) & =\frac{2 \mathscr{E}(r)-\left(1-r^{2}\right) \mathscr{K}(r)}{1+r} .
\end{aligned}
$$

Lemma 1 (see [17, Theorem 1.25]). Let $-\infty<a<b<$ $\infty, f, g:[a, b] \rightarrow(-\infty, \infty)$ be continuous on $[a, b]$ and differentiable on $(a, b)$, and $g^{\prime}(x) \neq 0$ on $(a, b)$. If $f^{\prime}(x) / g^{\prime}(x)$ is increasing (decreasing) on $(a, b)$, then so are

$$
\begin{aligned}
& \frac{f(x)-f(a)}{g(x)-g(a)}, \\
& \frac{f(x)-f(b)}{g(x)-g(b)} .
\end{aligned}
$$

If $f^{\prime}(x) / g^{\prime}(x)$ is strictly monotone, then the monotonicity in the conclusion is also strict.

Lemma 2 (see [17, Theorem 3.21]). (1) Function $r \mapsto[\mathscr{E}(r)-$ $\left.\left(1-r^{2}\right) \mathscr{K}(r)\right] / r^{2}$ is strictly increasing from $(0,1)$ to $(\pi / 4,1)$.

(2) Function $r \mapsto\left(1-r^{2}\right)^{\lambda} \mathscr{K}(r)$ is strictly decreasing from $(0,1)$ to $(0, \pi / 2)$ if $\lambda \geq 1 / 4$. 
Theorem 3. Double inequality

$$
\begin{gathered}
\lambda_{1} C(a, b)+\left(1-\lambda_{1}\right) A(a, b)<T(a, b) \\
<\mu_{1} C(a, b)+\left(1-\mu_{1}\right) A(a, b)
\end{gathered}
$$

holds for all $a, b>0$ with $a \neq b$ if and only if $\lambda_{1} \leq 1 / 8$ and $\mu_{1} \geq 4 / \pi-1=0.2732 \ldots$.

Proof. Since $A(a, b), T(a, b)$, and $C(a, b)$ are symmetric and homogeneous of degree 1 , without loss of generality, we assume that $a>b>0$. Let $r=(a-b) /(a+b) \in(0,1)$. Then (1) and (2) lead to

$$
\begin{aligned}
T(a, b) & =\frac{2 a}{\pi} \mathscr{E}\left(\frac{2 \sqrt{r}}{1+r}\right) \\
& =\frac{2 A(a, b)}{\pi}\left[2 \mathscr{E}(r)-\left(1-r^{2}\right) \mathscr{K}(r)\right], \\
C(a, b) & =A(a, b) \frac{1+3 r^{2}}{1+r^{2}} .
\end{aligned}
$$

We clearly see that inequality (16) is equivalent to

$$
\lambda_{1}<\frac{T(a, b)-A(a, b)}{C(a, b)-A(a, b)}<\mu_{1} .
$$

It follows from (17) and (18) that

$$
\begin{aligned}
& \frac{T(a, b)-A(a, b)}{C(a, b)-A(a, b)} \\
& \quad=\frac{1}{\pi} \frac{2 \mathscr{E}(r)-\left(1-r^{2}\right) \mathscr{K}(r)-\pi / 2}{r^{2} /\left(1+r^{2}\right)} .
\end{aligned}
$$

Let

$$
\begin{aligned}
f(r) & =\frac{2 \mathscr{E}(r)-\left(1-r^{2}\right) \mathscr{K}(r)-\pi / 2}{r^{2} /\left(1+r^{2}\right)}, \\
f_{1}(r) & =2 \mathscr{E}(r)-\left(1-r^{2}\right) \mathscr{K}(r)-\frac{\pi}{2}, \\
f_{2}(r) & =\frac{r^{2}}{1+r^{2}} .
\end{aligned}
$$

Then simple computations lead to

$$
\begin{aligned}
& f_{1}\left(0^{+}\right)=f_{2}\left(0^{+}\right)=0, \\
& \frac{f_{1}^{\prime}(r)}{f_{2}^{\prime}(r)}=\frac{\left(1+r^{2}\right)^{2}}{2} \frac{\mathscr{E}(r)-\left(1-r^{2}\right) \mathscr{K}(r)}{r^{2}} .
\end{aligned}
$$

From Lemmas 1 and 2 together with (21) and (22) we know that $f(r)$ is strictly increasing on $(0,1)$ and

$$
\begin{aligned}
& f\left(0^{+}\right)=\frac{f_{1}^{\prime}\left(0^{+}\right)}{f_{2}^{\prime}\left(0^{+}\right)}=\frac{\pi}{8}, \\
& f\left(1^{-}\right)=4-\pi .
\end{aligned}
$$

Therefore, Theorem 3 follows from (19)-(21) and (23) together with the monotonicity of $f(r)$.
Theorem 4. Let $\lambda_{2}, \mu_{2} \in(1 / 2,1)$. Then double inequality

$$
\begin{gathered}
C\left[\lambda_{2} a+\left(1-\lambda_{2}\right) b, \lambda_{2} b+\left(1-\lambda_{2}\right) a\right]<T(a, b) \\
<C\left[\mu_{2} a+\left(1-\mu_{2}\right) b, \mu_{2} b+\left(1-\mu_{2}\right) a\right]
\end{gathered}
$$

holds for all $a, b>0$ with $a \neq b$ if and only if $\lambda_{2} \leq 1 / 2+\sqrt{2} / 8=$ $0.6767 \ldots$ and $\mu_{2} \geq 1 / 2+\sqrt{(4-\pi) /(3 \pi-4)} / 2=0.6988 \ldots$

Proof. Let $\lambda_{2}^{*}=1 / 2+\sqrt{2} / 8$ and $\mu_{2}^{*}=1 / 2+$ $\sqrt{(4-\pi) /(3 \pi-4)} / 2$. We first prove that

$$
\begin{aligned}
& T(a, b)>C\left[\lambda_{2}^{*} a+\left(1-\lambda_{2}^{*}\right) b, \lambda_{2}^{*} b+\left(1-\lambda_{2}^{*}\right) a\right] \\
& T(a, b)<C\left[\mu_{2}^{*} a+\left(1-\mu_{2}^{*}\right) b, \mu_{2}^{*} b+\left(1-\mu_{2}^{*}\right) a\right]
\end{aligned}
$$

for all $a, b>0$ with $a \neq b$.

Without loss of generality, we assume that $a>b$. Let $r=$ $(a-b) /(a+b) \in(0,1)$ and $p \in(1 / 2,1)$. Then (2) leads to

$$
\begin{gathered}
C[p a+(1-p) b, p b+(1-p) a] \\
=A(a, b) \frac{3(2 p-1)^{2} r^{2}+1}{(2 p-1)^{2} r^{2}+1} .
\end{gathered}
$$

It follows from (17) and (27) that

$$
\begin{aligned}
& T(a, b)-C[p a+(1-p) b, p b+(1-p) a] \\
& \quad=A(a, b)\left[\frac{2}{\pi}\left(2 \mathscr{E}(r)-\left(1-r^{2}\right) \mathscr{K}(r)\right)\right. \\
& \left.\quad+\frac{2}{(2 p-1)^{2} r^{2}+1}-3\right] .
\end{aligned}
$$

Let

$$
\begin{aligned}
g(r)= & \frac{2}{\pi}\left(2 \mathscr{E}(r)-\left(1-r^{2}\right) \mathscr{K}(r)\right) \\
& +\frac{2}{(2 p-1)^{2} r^{2}+1}-3, \\
g_{1}(r)= & \frac{1}{r} g^{\prime}(r) .
\end{aligned}
$$


Then making use of Lemma 2 and simple computations lead to

$$
\begin{aligned}
g(0)= & 0, \\
g(1)= & \frac{4}{\pi}+\frac{2}{(2 p-1)^{2}+1}-3, \\
g_{1}(r)= & \frac{2}{\pi} \frac{\mathscr{E}(r)-\left(1-r^{2}\right) \mathscr{K}(r)}{r^{2}} \\
& -\frac{4(2 p-1)^{2}}{\left[(2 p-1)^{2} r^{2}+1\right]^{2}}, \\
g_{1}(0)= & \frac{1}{2}-4(2 p-1)^{2}, \\
g_{1}(1)= & \frac{2}{\pi}-\frac{4(2 p-1)^{2}}{\left[(2 p-1)^{2}+1\right]^{2}} .
\end{aligned}
$$

We divide the proof into two cases.

Case 1. Consider $p=\lambda_{2}^{*}=1 / 2+\sqrt{2} / 8$. Then (34) becomes

$$
g_{1}(0)=0 \text {. }
$$

It follows from Lemma 2(1) and (33) together with (36) that

$$
g_{1}(r)>0
$$

for all $r \in(0,1)$.

Therefore, inequality (25) follows easily from (28)-(31) and (37).

Case 2. Consider $p=\mu_{2}^{*}=1 / 2+\sqrt{(4-\pi) /(3 \pi-4)} / 2$. Then (32), (34), and (35) lead to

$$
\begin{aligned}
& g(1)=0 \\
& g_{1}(0)=-\frac{36-11 \pi}{6 \pi-8}<0, \\
& g_{1}(1)=\frac{3 \pi^{2}-14 \pi+16}{\pi^{2}}>0 .
\end{aligned}
$$

It follows from Lemma 2(1), (33), (39), and (40) that there exists $r_{0} \in(0,1)$ such that $g_{1}(r)<0$ for $r \in\left(0, r_{0}\right)$ and $g_{1}(r)>0$ for $r \in\left(r_{0}, 1\right)$. Then (30) leads to the conclusion that $g(r)$ is strictly decreasing on $\left(0, r_{0}\right]$ and strictly increasing on $\left(r_{0}, 1\right]$.

Therefore, inequality (26) follows easily from (28), (29), (31), (38), and the piecewise monotonicity of $g(r)$.

Next, we prove that $\lambda_{2}=\lambda_{2}^{*}=1 / 2+\sqrt{2} / 8$ is the best possible parameter on $(1 / 2,1)$ such that inequality

$$
T(a, b)>C\left[\lambda_{2} a+\left(1-\lambda_{2}\right) b, \lambda_{2} b+\left(1-\lambda_{2}\right) a\right]
$$

holds for all $a, b>0$ with $a \neq b$.

Indeed, if $\lambda_{2}^{*}=1 / 2+\sqrt{2} / 8<p<1$, then (34) leads to $g_{1}(0)<0$ and there exists $\delta_{1} \in(0,1)$ such that

$$
g_{1}(r)<0
$$

for $r \in\left(0, \delta_{1}\right)$.
Equations (28)-(31) and inequality (42) imply that

$$
T(a, b)<C[p a+(1-p) b, p b+(1-p) a]
$$

for $(a-b) /(a+b) \in\left(0, \delta_{1}\right)$.

Finally, we prove that $\mu_{2}=\mu_{2}^{*}=1 / 2+\sqrt{(4-\pi) /(3 \pi-4)} /$ 2 is the best possible parameter on $(1 / 2,1)$ such that double inequality

$$
T(a, b)<C\left[\mu_{2} a+\left(1-\mu_{2}\right) b, \mu_{2} b+\left(1-\mu_{2}\right) a\right]
$$

for all $a, b>0$ with $a \neq b$.

In fact, if $1 / 2<p<\mu_{2}^{*}=1 / 2+\sqrt{(4-\pi) /(3 \pi-4)} / 2$, then (32) leads to $g(1)>0$ and there exists $\delta_{2} \in(0,1)$ such that

$$
g(r)>0
$$

for $r \in\left(1-\delta_{2}, 1\right)$.

Equations (28) and (29) together with inequality (45) imply that

$$
T(a, b)>C[p a+(1-p) b, p b+(1-p) a]
$$

for $(a-b) /(a+b) \in\left(1-\delta_{2}, 1\right)$.

Let $r \in(0,1), a=1, b=\sqrt{1-r^{2}}, \lambda_{1}=1 / 8, \mu_{1}=4 / \pi-1$, $\lambda_{2}=1 / 2+\sqrt{2} / 8$, and $\mu_{2}=1 / 2+\sqrt{(4-\pi) /(3 \pi-4)} / 2$. Then Theorems 3 and 4 lead to Corollary 5 as follows.

\section{Corollary 5. Double inequalities}

$$
\begin{aligned}
& \frac{\pi\left[1+\left(1-r^{2}\right)^{3 / 2}\right]}{16\left(2-r^{2}\right)}+\frac{7 \pi\left[1+\left(1-r^{2}\right)^{1 / 2}\right]}{32}<\mathscr{E}(r) \\
& <\frac{(4-\pi)\left[1+\left(1-r^{2}\right)^{3 / 2}\right]}{2\left(2-r^{2}\right)}+\left(\frac{\pi}{2}-1\right)\left[1+\left(1-r^{2}\right)^{1 / 2}\right], \\
& \frac{\pi}{2} \frac{32-21 r^{2}+21\left(1-r^{2}\right)^{1 / 2}+11\left(1-r^{2}\right)^{3 / 2}}{36-18 r^{2}+28\left(1-r^{2}\right)^{1 / 2}}<\mathscr{E}(r)<\frac{\pi}{2} \\
& \cdot \frac{3 \pi-4-3(\pi-2) r^{2}+3(\pi-2)\left(1-r^{2}\right)^{1 / 2}+2\left(1-r^{2}\right)^{3 / 2}}{\pi\left(2-r^{2}\right)+4(\pi-2)\left(1-r^{2}\right)^{1 / 2}}
\end{aligned}
$$

hold for all $r \in(0,1)$.

\section{Conflict of Interests}

The authors declare that there is no conflict of interests regarding the publication of this paper.

\section{Acknowledgments}

This research was supported by the Natural Science Foundation of China under Grants 11371125, 11401191, and 61374086, the Natural Science Foundation of Zhejiang Province under Grant LY13A010004, the Natural Science Foundation of Hunan Province under Grant 12C0577, and the Natural Science Foundation of the Zhejiang Broadcast and TV University under Grant XKT-15G17. 


\section{References}

[1] G. Toader, "Some mean values related to the arithmeticgeometric mean," Journal of Mathematical Analysis and Applications, vol. 218, no. 2, pp. 358-368, 1998.

[2] E. Neuman, "Bounds for symmetric elliptic integrals," Journal of Approximation Theory, vol. 122, no. 2, pp. 249-259, 2003.

[3] H. Kazi and E. Neuman, "Inequalities and bounds for elliptic integrals," Journal of Approximation Theory, vol. 146, no. 2, pp. 212-226, 2007.

[4] H. Kazi and E. Neuman, "Inequalities and bounds for elliptic integrals II," in Special Functions and Orthogonal Polynomials, Contemporary Mathematics 471, pp. 127-138, American Mathematical Society, Providence, RI, USA, 2008.

[5] Y.-M. Chu, M.-K. Wang, and X.-Y. Ma, "Sharp bounds for Toader mean in terms of contraharmonic mean with applications," Journal of Mathematical Inequalities, vol. 7, no. 2, pp. 161166, 2013.

[6] Y.-Q. Song, W.-D. Jiang, Y.-M. Chu, and D.-D. Yan, "Optimal bounds for Toader mean in terms of arithmetic and contraharmonic means," Journal of Mathematical Inequalities, vol. 7, no. 4, pp. 751-757, 2013.

[7] W.-H. Li and M.-M. Zheng, "Some inequalities for bounding Toader mean," Journal of Function Spaces and Applications, vol. 2013, Article ID 394194, 5 pages, 2013.

[8] Y. Hua and F. Qi, "A double inequality for bounding Toader mean by the centroidal mean," Proceedings-Mathematical Sciences, vol. 124, no. 4, pp. 527-531, 2014.

[9] Y. Hua and F. Qi, "The best bounds for Toader mean in terms of the centroidal and arithmetic means," Filomat, vol. 28, no. 4, pp. 775-780, 2014.

[10] M. Vuorinen, "Hypergeometric functions in geometric function theory," in Special Functions and Differential Equations (Madras, 1977), pp. 119-126, Allied Publishers, New Delhi, India, 1998.

[11] S.-L. Qiu and J.-M. Shen, "On two problems concerning means," Journal of Hangzhou Institute of Electronic Engineering, vol. 17, no. 3, pp. 1-7, 1997 (Chinese).

[12] R. W. Barnard, K. Pearce, and K. C. Richards, "An inequality involving the generalized hypergeometric function and the arc length of an ellipse," SIAM Journal on Mathematical Analysis, vol. 31, no. 3, pp. 693-699, 2000.

[13] H. Alzer and S.-L. Qiu, "Monotonicity theorems and inequalities for the complete elliptic integrals," Journal of Computational and Applied Mathematics, vol. 172, no. 2, pp. 289-312, 2004.

[14] Y.-M. Chu, M.-K. Wang, S.-L. Qiu, and Y.-F. Qiu, "Sharp generalized Seiffert mean bounds for Toader mean," Abstract and Applied Analysis, vol. 2011, Article ID 605259, 8 pages, 2011.

[15] Y.-M. Chu and M.-K. Wang, "Inequalities between arithmeticgeometric, Gini, and Toader means," Abstract and Applied Analysis, vol. 2012, Article ID 830585, 11 pages, 2012.

[16] Y.-M. Chu and M.-K. Wang, "Optimal Lehmer mean bounds for the Toader mean," Results in Mathematics, vol. 61, no. 3-4, pp. 223-229, 2012.

[17] G. D. Anderson, M. K. Vamanamurthy, and M. K. Vuorinen, Conformal Invariants, Inequalities, and Quasiconformal Maps, John Wiley \& Sons, New York, NY, USA, 1997. 


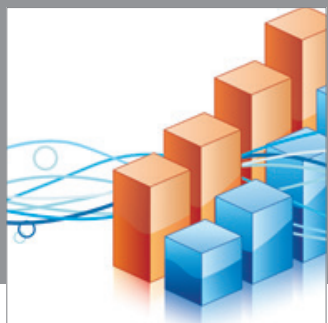

Advances in

Operations Research

mansans

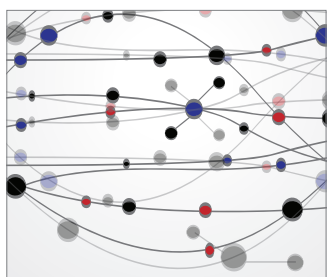

The Scientific World Journal
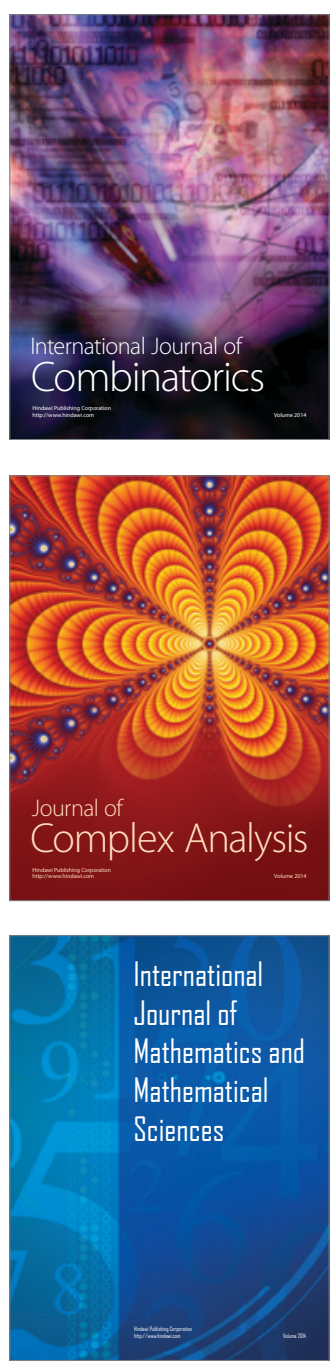
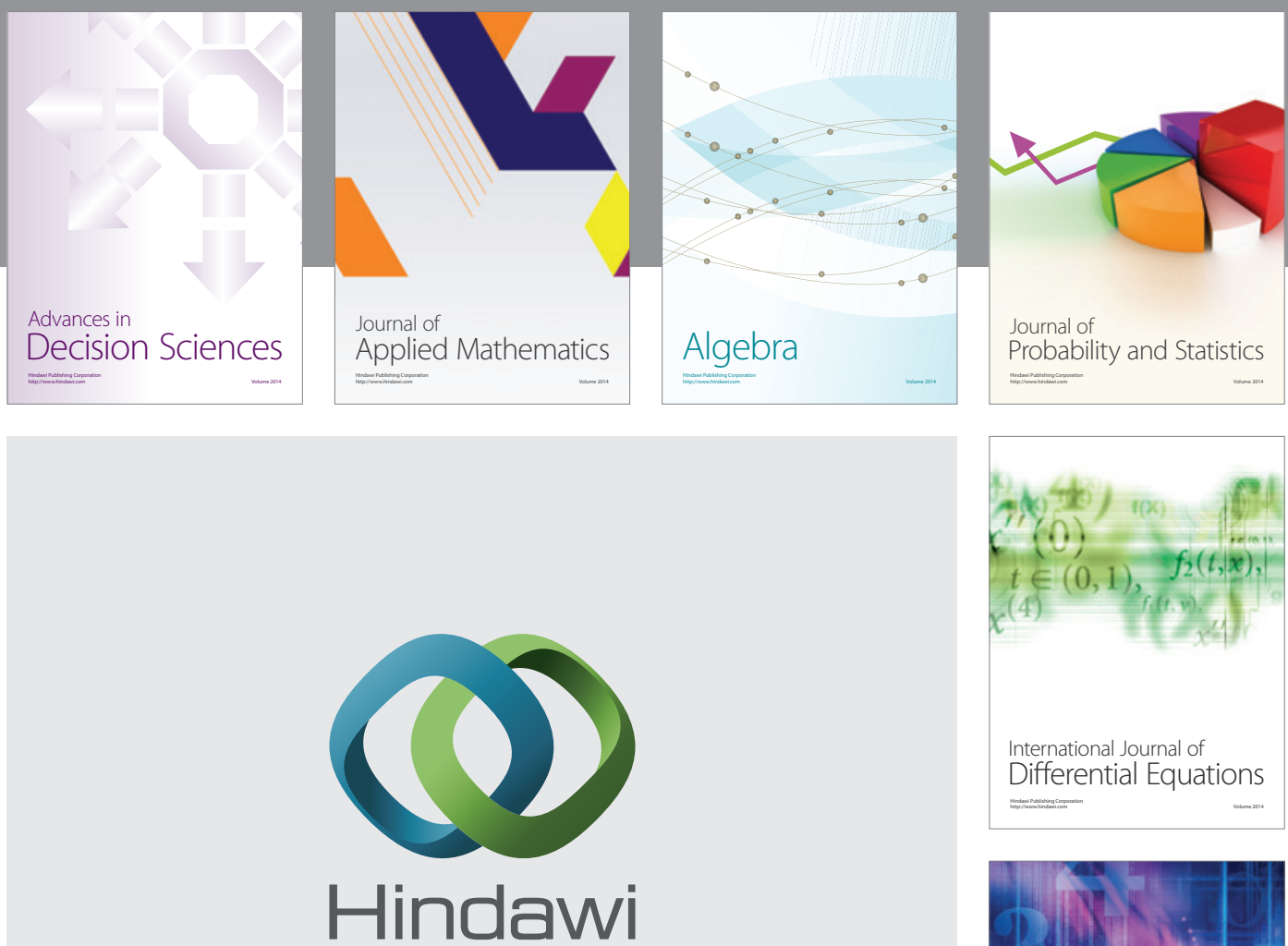

Submit your manuscripts at http://www.hindawi.com
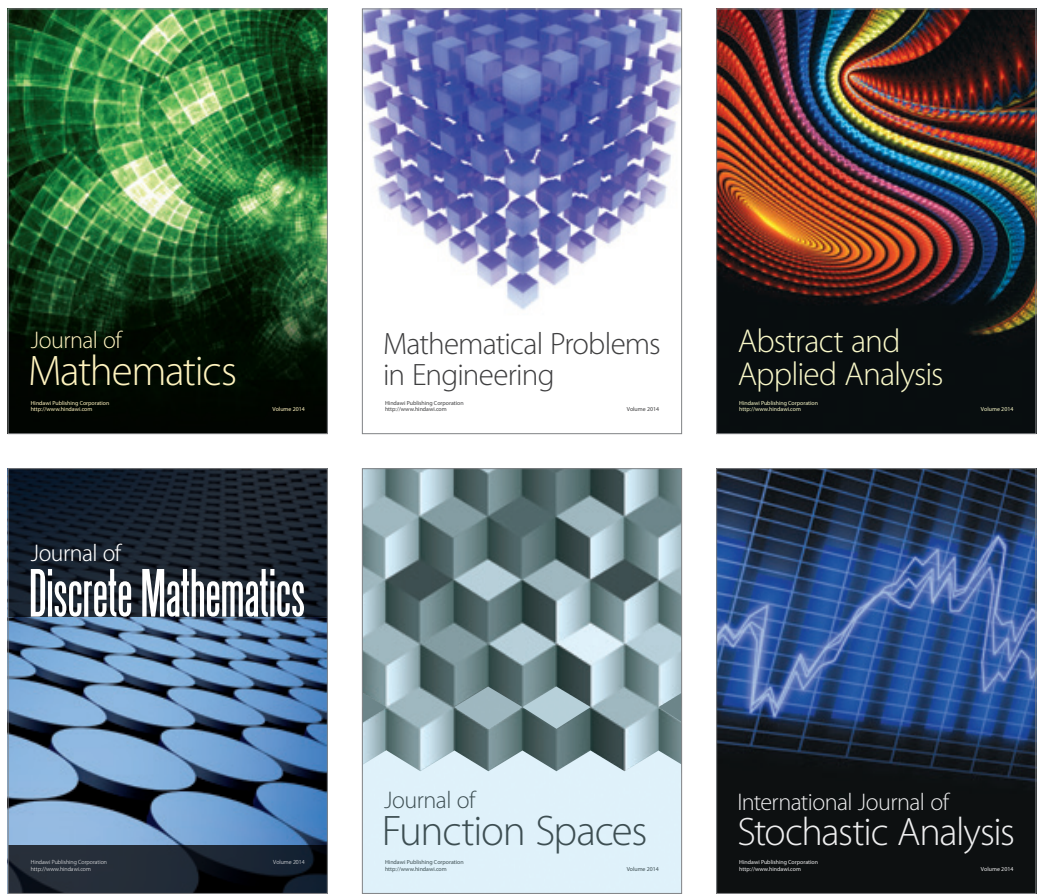

Journal of

Function Spaces

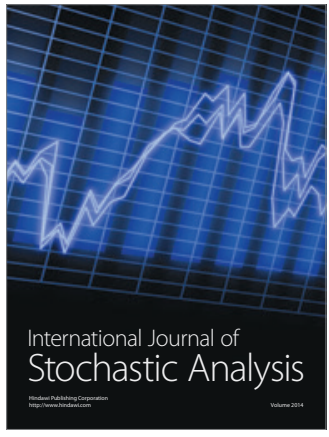

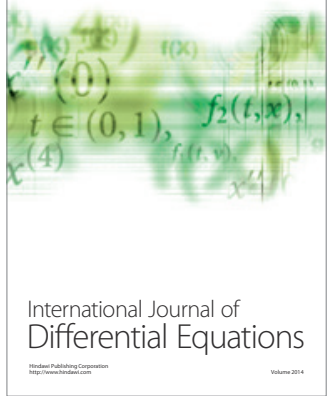
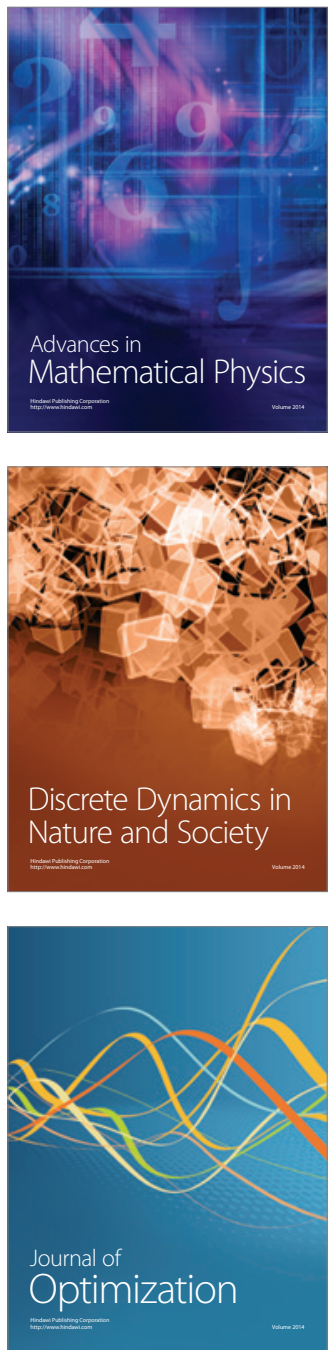